Pacific Journal of Mathematics

SPACES WITHOUT REMOTE POINTS

ic Karel van Douwen and Jan van MilL 


\title{
SPACES WITHOUT REMOTE POINTS
}

\author{
ERIC K. VAN Douwen and Jan VAN Mill
}

\begin{abstract}
All spaces considered are completely regular and $X^{*}$ denotes $\beta X-$ $X$. The point $x \in X^{*}$ is called a remote point of $X$ if $x \notin \mathrm{Cl}_{\beta X} A$ for each nowhere dense subset $A$ of $X$. If $y \in Y$, then the space $Y$ is said to be extremally disconnected at $y$ if $y \notin \bar{U} \cap \bar{V}$ whenever $U$ and $V$ are disjoint open sets. In this paper we construct two noncompact $\sigma$-compact spaces $X$, one locally compact and one nowhere locally compact, such that $X$ has no remote points, and in fact such that $\beta X$ is not extremally disconnected at any point.
\end{abstract}

Our examples were motivated by the following results from [6]:

(1) $X$ has remote points if $X$ has countable $\pi$-weight, in particular if $X$ is separable and first countable, and is not pseudocompact, $[6,1.5]$; see also [7] for an earlier consistency result, and [1] for a more general result.

(2) $\beta X$ is extremally disconnected at each remote point of $X,[6,5.2]$. Via the observation that

(3) if $Y$ is dense in $Z$, and $y \in Y$, then $Y$ is extremally disconnected at $y$ iff $Z$ is extremally disconnected at $y$,

these results and the following imply a nonhomogeneity result, which applies for example to the rationals and the Sorgenfrey line

(4) if $X$ is a nowhere locally compact nonpseudocompact space which has a remote point and if $\{x \in X: X$ is not extremally disconnected at $x\}$ is dense in $X$, e.g. if $X$ is first countable, then $X^{*}$ is not homogeneous because $X^{*}$ is extremally disconnected at some but not at all points.

(This is a special case of Frolík's theorem that $X^{*}$ is not homogeneous if $X$ is not pseudocompact, [8]. The proof of Frolik's theorem does not yield a simple "because" as in (4). $X$ is called nowhere locally compact if no point of $X$ has a compact neighborhood, or, equivalently, if $X^{*}$ is dense in $\beta X$.)

In this paper we produce two closely related examples which show that the condition on the $\pi$-weight cannot be omitted altogether in (1), thus answering a question of [6].

Our two examples are rather big: they have cellularity at least $\omega_{3}$. This suggests the question of whether every nonpseudocompact separable space has a remote point. (This would generalize (1).) It follows from a construction in [7] that the answer is affirmative under $\mathrm{CH}$. 
EXAMPLES. There are two noncompact $\sigma$-compact spaces $X$, one locally compact and one nowhere locally compact, such that $X$ has no remote points, and in fact such that $\beta X$ is not extremally disconnected at any point.

Because of (3) the nowhere locally compact example shows that the condition on the $\pi$-weight cannot be omitted altogether in the nonhomogeneity result (4). We will show that an older nonhomogeneity proof, involving far points, still applies.

\section{No remote points.}

A subset $P$ of a space $X$ is called a $P$-set if for each $F_{\sigma}$-subset $F$ of $X$, if $F \cap P=\varnothing$ then $\bar{F} \cap P=\varnothing$. A subset $T$ of a space $X$ is called a 2-set if there are disjoint open $U$ and $V$ in $X$ with $T \subseteq \bar{U} \cap \bar{V}$.

LEMMA 1. There is a compact space $U$ such that for each $q \in U$ there is a decreasing $\omega_{1}$-sequence $\left\langle P_{\xi}: \xi \in \omega_{1}\right\rangle$ of clopen sets such that $\bigcap_{\xi \in \omega_{1}} P_{\xi}$ is a nowhere dense set of $U$ which contains $q$.

$\square$ Give $\omega_{2}$ the discrete topology. Identify $\omega_{2}^{*}$ with the space of free ultrafilters on $\omega_{2}$. Then

$$
U=\left\{q \in \omega_{2}^{*}:|Q|=\omega_{2} \text { for all } Q \in q\right\},
$$

the space of uniform ultrafilters on $\omega_{2}$, is a closed, hence compact, subspace of $\omega_{2}^{*}$ of course. We need the following result due to Čudnovskiı̆ and Čudnovskiĭ, [3] and, independently, to Kuen and Prikry, [11], and earlier, but with GCH to Chang [2]:

for each $q \in U$ there is a decreasing $\omega_{1}$-sequence $\left\langle Q_{\xi}: \xi \in \omega_{1}\right\rangle$ in (*) $\quad q$ such that $\bigcap_{\xi \in \omega_{1}} Q_{\xi}=\varnothing$.

As usual, let $\hat{A}$ denote $U \cap \bar{A}$ (closure in $\beta \omega_{2}$ ), for $A \subseteq \omega_{2}$. For a given $q \in U$ let $\left\langle Q_{\xi}: \xi \in \omega_{1}\right\rangle$ be as in $(*)$, and define $\left\langle P_{\xi}: \xi \in \omega_{1}\right\rangle$ by $P_{\xi}=\hat{Q}_{\xi}$ for $\xi \in \omega_{1}$. Clearly $\left\langle P_{\xi}\right.$ : $\left.\xi \in \omega_{1}\right\rangle$ is a decreasing $\omega_{1}$-sequence of clopen subsets of $U$ such that $P=\bigcap_{\xi \in \omega_{1}} P_{\xi}$ contains $q$. Now recall that $\{\hat{B}$ : $B \subseteq \omega_{2}$ and $\left.|B|=\omega_{2}\right\}$, being the collection of all nonempty clopen subsets of $U$, is a base for $U$. Consider any $B \subseteq \omega_{2}$ with $|B|=\omega_{2}$. There is an $\eta \in \omega_{1}$ with $\left|B-Q_{\eta}\right|=\omega_{2}$. Then $\varnothing \neq\left(B-Q_{\eta}\right)=\hat{B}-\hat{Q}_{\eta} \subseteq \hat{B}-P$. It follows that $P$ is nowhere dense.

REMARK. Instead of $\omega_{1}$ we can take any regular cardinal $\kappa$, and then $U$ will be the space of uniform ultrafilters on $\kappa^{+}$. 
Clearly Lemma 1 implies that there is a compact space which is covered by the collection of its nowhere dense closed $P$-sets. Since evidently each 2-set is nowhere dense the following is a stronger assertion.

LEMMA 2. There is a compact space $H$ such that for each $q \in H$ there is a closed $P$ in $H$ with $q \in P$ such that $P$ is both a P-set and a 2-set.

Let $U$ be as in Lemma 1, and let $H=U \times U$. Consider any $q_{0}, q_{1} \in U$. For $i \in 2$ choose a decreasing $\omega_{1}$-sequence $\left\langle P_{i, \xi}: \xi \in \omega_{1}\right\rangle$ of clopen sets in $U$ such that $P_{1}=\bigcap_{\xi \in \omega_{1}} P_{i, \xi}$ is a nowhere dense subset of $U$ which contains $q_{l}$. Then $P_{0} \times P_{1}$ is a nowhere $P$-set in $H$ which contains $\left\langle q_{0}, q_{1}\right\rangle$. We show that $P_{0} \times P_{1}$ is also a 2 -set

For $i \in 2$ define an open $V_{l, \xi}$ with recursion on $\xi \in \omega_{1}$ by

$$
V_{i, \xi}=\left(U-P_{l, \xi}\right)-\left(\bigcup_{\eta \in \xi} V_{i, \eta}\right)^{-} \quad\left(\bigcup_{\nu \in 0} V_{i, \eta}=\varnothing \text { of course }\right) .
$$

Then evidently $\left(\cup_{\eta \leq \xi} V_{t, \eta}\right)^{-}=U-P_{t, \xi}$ for $i \in 2$ and $\xi \in \omega_{1}$. Since $P_{0}$ and $P_{1}$ are nowhere dense it follows that

$$
\left(\bigcup_{\xi \in \omega_{1}} V_{l, \xi}\right)^{-}=\left(U-P_{i}\right)^{-}=U, \quad \text { for } i \in 2 .
$$

Define open subsets $W_{0}$ and $W_{1}$ of $H$ by

$$
W_{0}=\bigcup_{\xi \in \omega_{1}} P_{0, \xi} \times V_{1, \xi} \quad \text { and } \quad W_{1}=\bigcup_{\xi \in \omega_{1}} V_{0, \xi} \times P_{1, \xi} .
$$

Then $W_{0} \cap W_{1}=\varnothing$ since if $\xi \leq \eta<\omega_{1}$ then $V_{i, \xi} \subseteq U-P_{l, \xi} \subseteq U-P_{i, \eta}$, for $i \in 2$ (so that $\left(P_{0, \xi} \times V_{1, \xi}\right) \cap\left(V_{0, \eta} \times P_{1, \xi}\right)=\varnothing$ for all $\left.\xi, \eta \in \omega_{1}\right)$. To prove that $P_{0} \times P_{1} \subseteq \bar{W}_{0} \cap \bar{W}_{1}$ we have only to prove that $P_{0} \times P_{1} \subseteq \bar{W}_{0}$, because of symmetry. We have

$$
W_{0} \supseteq \bigcup_{\xi \in \omega_{1}}\left(\left(\bigcap_{\eta \in \omega_{1}} P_{0, \eta}\right) \times V_{1, \xi}\right)=P_{0} \times \bigcup_{\xi \in \omega_{1}} V_{1, \xi},
$$

hence $\bar{W}_{0} \supseteq P_{0} \times U \supseteq P_{0} \times P_{1}$ as required.

REMARK 2. We do not know if the space $U$ of Lemma 1 can be used for the space $H$ of Lemma 2. We are indebted to the referee for pointing out that the set $P=\bigcap_{\xi \in \omega_{1}} P_{\xi}$ obtained in Lemma 1 is not a 2-set: $P$ has character $\omega_{1}$, but in $U$ the closure of every open $F_{\omega_{1}}$-set ( $\equiv$ union of $\omega_{1}$ many closed sets) is easily seen to be open, [CoN, Thm. 14.9], which implies that no closed set in $U$ of character $\omega_{1}$ is a 2 -set. To see this let $F$ be a closed set in $U$ of character $\omega_{1}$ and let $V$ and $W$ be disjoint open sets 
in $U$ such that $F \subseteq \bar{V}$. Since $F$ has character $\omega_{1}$ there is an open $F_{\omega_{1}}$-set $T \subseteq V$ such that $\bar{T} \cap \bar{F} \neq \varnothing$. Now $\bar{T} \cap W=\varnothing$ since $T \cap W=\varnothing$, and $\bar{T}$ is clopen. It follows that $F \nsubseteq \bar{W}$.

SUBREMARK. It is at least consistent that $U=U\left(\omega_{2}\right)$ has a closed $P$-set that is a 2-set. There is a closed nowhere dense $P \subseteq U$ which is a $P_{\omega_{2}}$-set ( $\equiv$ for every $F_{\omega_{2}}$-set $F$ in $U$, if $F \cap P=\varnothing$ then $\bar{F} \cap P=\varnothing$ ), namely $\cap\left\{C: C \subseteq \omega_{2}\right.$ is a cub $\}$, and if $2^{\omega_{2}}=\omega_{3}$ then every nowhere dense $P_{\omega_{3}}$-set in $U$ (or in any space of weight $\omega_{3}$ ) is a 2-set. However, if $2^{\omega_{2}}=\omega_{3}$ then $U$ is not covered by the collection of its nowhere dense closed $P_{\omega_{3}}$-sets, by $[10,1.1]$.

REMARK 3. After this paper had been written another proof of Lemma 1 was discovered by Kunen, van Mill and Mills: the space of nondecreasing functions $\omega_{2} \rightarrow \omega_{1}+1,[10,3.1]$. It is easy to see that the $P$-sets obtained there are 2-sets. The example of Lemma 2 has the additional feature that each $P$-set has character $\omega_{1}$.

REMARK 4. The above remarks suggest the question of whether there is a compact space which is covered by the collection of its closed nowhere dense $P$-sets but which has no nonempty closed $P$-set which is also a 2 -set. This question can be answered quite easily. Let $E$ be the projective cover of the example of Lemma 1, i.e. $E$ is the unique extremally disconnected compact space that admits an irreducible map, say $\pi$, onto $U$. As is well known, $\pi^{\leftarrow}(D)$ is nowhere dense in $E$ iff $D$ is nowhere dense in $E$. Since it is easily seen that $\pi^{-}(P)$ is a $P$-set of $E$ iff $P$ is a $P$-set of $U$, we conclude that $E$ can be covered by nowhere dense closed $P$-sets. Since $E$ is extremally disconnected, there are no nonempty 2 -sets in $E$. The following question however remains open:

Question. Is there (in ZFC) a compact space which is covered by the collection of its closed nowhere dense $P$-sets but which has no nonempty nowhere dense $P_{\omega_{2}}$-set?

Lemma 3. Let $K$ be a compact space, and let $P$ be a $P$-set in $K$. Furthermore, let $Y$ be a countable space, let $\pi: K \times Y \rightarrow K$ be the projection, and let $\beta \pi: \beta(K \times Y) \rightarrow K$ be the Stone extension of $\pi$. Then for each $x \in \beta(K \times Y)$, if $\beta \pi(x) \in P$ then $x \in(P \times Y)^{-}$.

Consider any $x \in \beta(K \times Y)-(P \times Y)^{-}$. Let $V$ be a closed neighborhood of $x$ which misses $P \times Y$. Then $x \in((K \times Y) \cap V)^{-}$, hence

$$
\beta \pi(x) \in\left(\beta \pi^{\rightarrow}((K \times Y) \cap V)\right)^{-}=\left(\pi^{\rightarrow}((K \times Y) \cap V)\right)^{-} .
$$


Also, $\pi^{\rightarrow}((K \times Y) \cap V)$ is an $F_{\sigma}$ (since $(K \times Y) \cap V$ is $\sigma$-compact) in $K$ which misses the $P$-set $P$, hence $\left(\pi^{\rightarrow}((K \times Y) \cap V)\right)^{-} \cap P=\varnothing$. Consequently $\beta \pi(x) \notin P$.

COROLlary 1. If $K$ is a compact space which is covered by nowhere dense $P$-sets, then $K \times Y$ has no remote points, for each countable space $Y$.

COROLlaRY 2. If $K$ is a compact space which is covered by P-sets which are 2-sets, then $\beta(K \times Y)$ is not extremally disconnected at any point, for each countable space $K$.

$\square$ The key observation is that if $D$ is dense in a space $X$, then the closure in $X$ of each 2-set in $D$ is a 2-set in $X$.

If $H$ is as in Lemma 2, if $\omega$ is the integers and if $Q$ is the rationals, then our examples are $H \times \omega$ and $H \times Q$.

Far points.

A point $p$ of $X^{*}$ is called a far (or $\omega$-far) point of $X$ if $p \notin \mathrm{Cl}_{\beta X} D$ for each (countable) closed discrete subset $D$ of $X$. Clearly, if $X$ has no isolated points then each remote point of $X$ is a far point; the converse of this is generally false, $[6,4.8]$. There is a nonhomogeneity result involving far points, or $\omega$-far points, similar to (4) of the introduction, but less attractive since it involves $X^{* *}=\left(X^{*}\right)^{*}$ : If $X$ is nowhere locally compact, and is not countably compact, and has a far ( $\omega$-far) point, then $X^{*}$ is not homogeneous because for some but not for all $x \in X^{*}$ there is a (countable) closed discrete $D$ in the space $X^{* *}$ such that $x \in \mathrm{Cl}_{\beta X^{*}} D,[5,2,4.3]$.

One might hope that our examples can be used to answer the question of [5] of whether every noncompact Lindelöf space has an $\omega$-far point (which would be a far point). (It is easy to see that every normal nonLindelöf space has an $\omega$-far point, $[\mathbf{5}, 4.3]$.) This is not the case: both our examples have far points. This follows from the following result.

THEOREM. If $X$ has a countably infinite discrete collection $K$ of compact subspaces without isolated points, and if $X$ is normal, or, more generally, if $K$ can be separated by a discrete open family, then $X$ has a far point.

Before we proceed to the proof we point out an attractive corollary:

COROLlARY. Every locally compact (or, more generally, ¿̌ech-complete) nonpseudocompact space has a far-point. 
If $X$ is nonpseudocompact it has a countably infinite family $\mathcal{Q}$ consisting of nonempty open sets. By a well-known tree argument one finds for each $U \in \mathcal{Q}$ a compact $K_{U} \subseteq U$ that admits a continuous map $f_{U}$ onto the Cantor discontinuum ${ }^{\omega} 2$. For $U \in \mathcal{Q}$ choose a compact $L_{U} \subseteq K_{U}$ such that $f_{U} \backslash L_{U}$ is an irreducible map onto ${ }^{\omega} 2$, then $L_{U}$ has no isolated points.

Proof of Theorem. First recall that $\mathbf{R}$ has a far point, by an elegant argument due to Eberlein [7, Thm. 1.3]. It follows that $Y=U \mathcal{K}$ has a far point. As in the proof of the Corollary, each member of $\mathcal{K}$ admits a (necessarily closed) map onto the Cantor discontinuum, hence on the closed unit interval. Since $\mathcal{K}$ is countably infinite it follows that $Y$ admits a closed map onto $\mathbf{R}$. The Stone extension $\beta f$ of $f$ maps $\phi Y$ onto $\beta \mathbf{R}$, hence there is $y \in Y^{*}$ such that $\beta f(y)$ is a far point of $R$. Since $f^{\rightarrow} D$ is closed discrete in $\mathbf{R}$ for each closed discrete $D$ in $Y$ this $y$ is a far point of $Y$, cf. [5, §2, Fact 3].

We now point out that

For any two disjoint closed $F$ and $G$ in $X$, if $F \subseteq Y$ then

$$
\mathrm{Cl}_{\beta X} F \cap \mathrm{Cl}_{\beta X} G=\varnothing \text {. }
$$

The proof is similar to the known case, $[9,3 \mathrm{~L}]$, that $\mathcal{K}$ consists of singletons. From (*) we see that $\mathrm{Cl}_{\beta X} Y=\beta Y$. Since $Y$ is closed in $X$ it follows that $X$ contains a far point of $Y$. This point is a far point of $X$ since, by (*), for each closed discrete subset $D$ of $Y$ we have $\mathrm{Cl}_{\beta X}(D-Y)$ $\cap \mathrm{Cl}_{\beta X} Y=\varnothing$.

REMARK 5. Dow [4] has shown that every separable nonpseudocompact space has a remote point under MA.

Remark 6. After this paper was written there has been much progress on the question of whether every Lindelöf space has a far point: It is known that the answer is affirmative under MA, [12,9.1].

\section{REFERENCES}

1. S. B. Chae and J. H. Smith, Remote points and G-spaces, Topology Appl., 1 (1980), 243-246.

2. C. C. Chang, Descendingly incomplete ultrafilters, Trans. Amer. Math. Soc., 126 (1967), $108-118$.

3. G. B. Čudnovskiī and D. D. Čudnovskiı̄, Regular and descending ultrafilters, Sov. Math. Dokl., 12 (1971), 901-905.

4. A. Dow, Weak P-points in compact ccc F-spaces, to appear in Trans. Amer. Math. Soc., 5. E. K. van Douwen, Why certain Čech-Stone remainders are not homogeneous, Coll. Math., 41 (1979), 45-52.

6. , Remote points, Diss. Math., 188 (1980). 
7. N. J. Fine and L. Gillman, Remote points in $\beta R$, Proc. Amer. Math. Soc., 13 (1962), 29-36.

8. Z. Frolik, Non-homogeneity of $\beta P-P$, Comm. Math. Univ. Car., 8 (1967), 705-709.

9. L. Gillman and M. Jerison, Rings of continuous functions, Princeton, van Nostrand, 1960.

10. K. Kunen, J. van Mill and C. F. Mills, On nowhere dense closed P-sets, Proc. Amer. Math. Soc., 78 (1980), 119-123.

11. K. Kunen and K. Prikry, On descendingly incomplete ultrafilters, J. Symbolic Logic, 36 (1971), 650-652.

12. J. van Mill, Weak P-points in $\check{C}$ ech-Stone compactifications, to appear in Trans. Amer. Math. Soc.

Received April 11, 1978 and in revised form March 10, 1982. Research of the first author was supported by an NSF grant, and the second author's research was supported by the Netherlands Organization for the Advancement of Pure Research Z.W.O.): Juliana van Stolberglaan 148, 's-Gravenhage, the Netherlands.

OHIO UNIVERSITY

ATHENS, OH 45701

AND

VRIJE UNIVERSITEIT

DE BOELELAAN 1081

$1081 \mathrm{HV}$, THE NeTHERLANDS

Current address of van Douwen: University of Wisconsin Madison, WI 53706 



\section{PACIFIC JOURNAL OF MATHEMATICS}

EDITORS

Donald BABBITT (Managing Editor)

University of California

Los Angeles, CA 90024

Hugo Rossi

University of Utah

Salt Lake City, UT 84112

C. C. Moore and Arthur Ogus

University of California

Berkeley, CA 94720
J. DugunduI

Department of Mathematics

University of Southern California

Los Angeles, CA 90089-1113

R. FinN and H. SAmelson

Stanford University

Stanford, CA 94305

\section{ASSOCIATE EDITORS}
R. ARENS
E. F. BECKENBACH
B. H. NEUMANN
F. WolF
K. YoshidA (1906-1982)

\section{SUPPORTING INSTITUTIONS}

UNIVERSITY OF ARIZONA

UNIVERSITY OF BRITISH COLUMBIA

CALIFORNIA INSTITUTE OF TECHNOLOGY

UNIVERSITY OF CALIFORNIA

MONTANA STATE UNIVERSITY

UNIVERSITY OF NEVADA, RENO

NEW MEXICO STATE UNIVERSITY

OREGON STATE UNIVERSITY
UNIVERSITY OF OREGON

UNIVERSITY OF SOUTHERN CALIFORNIA

STANFORD UNIVERSITY

UNIVERSITY OF HAWAII

UNIVERSITY OF TOKYO

UNIVERSITY OF UTAH

WASHINGTON STATE UNIVERSITY

UNIVERSITY OF WASHINGTON 


\section{Pacific Journal of Mathematics}

Vol. 105, No. 1 September, 1983

Kenneth F. Andersen, On the transformation of Fourier coefficients of

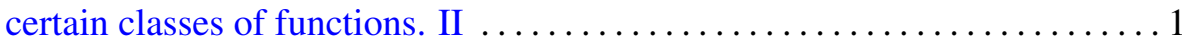

Gavin Brown, Irving Leonard Glicksberg and Edwin Hewitt, Indicator functions with large Fourier transforms $\ldots \ldots \ldots \ldots \ldots \ldots \ldots \ldots \ldots \ldots$

Shih-Sen Chang, Some random fixed point theorems for continuous random

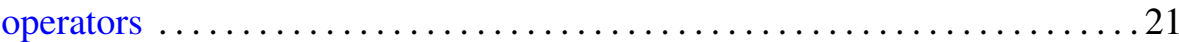

K. C. Chattopadhyay and Olav Njstad, Quasiregular nearness spaces and

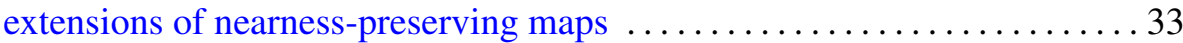

Thomas W. Cusick, The two-dimensional Diophantine approximation

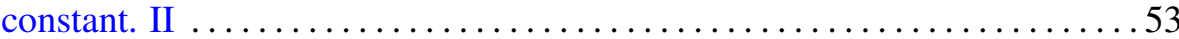

Eric Karel van Douwen and Jan van Mill, Spaces without remote points . . .669 Hector O. Fattorini, Convergence and approximation theorems for

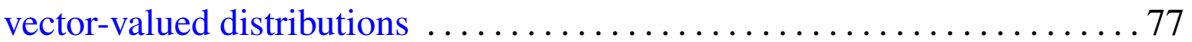

John J. F. Fournier and Louis Pigno, Analytic and arithmetic properties of

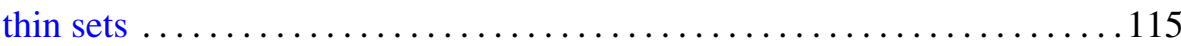

David Goss, On a new type of $L$-function for algebraic curves over finite

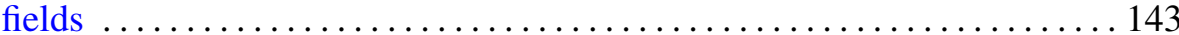

Douglas Austin Hensley, Lattice vertex polytopes with interior lattice

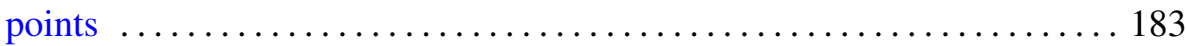

J. K. Kohli, Monotone extensions of mappings and their applications ...... 193

John C. Morgan, II, On equivalent category bases . . . . . . . . . . . . 207

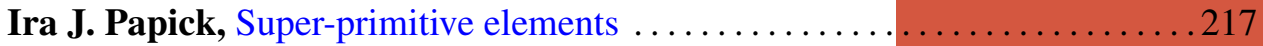

José Luis Rubio de Francia and José Luis Torrea, Vector extensions of

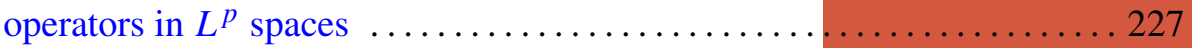

Mark Phillip Thomas, Closed ideals of $l^{1}\left(\omega_{n}\right)$ when $\left\{\omega_{n}\right\}$ is star-shaped $\ldots 237$ 